\title{
Design of Modified Smith predictor for dead time compensation for SOPDT process
}

\author{
Swati Singh,Mayank chaturvedi \& Pradeep K. Juneja \\ Graphic Era University,Dehradun,India
}

ABSTRACT: process industry many time used second order plus dead time process as an industrial process. Time taken between input and output of a phenomena in an industry is called dead time also known as transportation lag. Smith predictor is a dead time compensation technique which is used to compensate dead time. Smith predictor compare process model and actual process of the system until error between them zero, but there are some limitations of smith predictor if actual process and process model is not match. Modified Smith predictor is used to achieve stability and zero steady state error to step load response.

\section{INTRODUCTION}

In this paper we present a time delay compensation technique which deal with problematic area in process control industries-occurrence of time delay [1]. Time delay is also known as dead time, transportation lag. An interval during which an actuating signal produces no response known as dead time $[1,5]$. Smith predictor is the dead time compensation technique which is used to compensate the dead time but there are some draw back if actual process and model output is not match [7]. Here we use modified Smith predictor to compensate the dead time.

The dynamics of most of the industrial processes is modeled by second order plus dead time (SOPDT) transfer function. A number of control problems in the process industry are solved using PID controllers because of several reasons-maintenance and operation of PID controllers is simple and also they are robust in nature [3]. Tuning rule for PID controller is less for SOPDT as compare to FOPDT [6].

In the present analysis second order plus dead time is consider. The representation of second order plus dead time is

$$
G(s)=\frac{k}{\left(\tau_{1}+1\right)\left(\tau_{2}+1\right)} e^{-\theta s}
$$

Where $\mathrm{k}$ is the gain of the process $\theta$ is the delay time, $\tau 1$ and $\tau 2$ are the time constant.

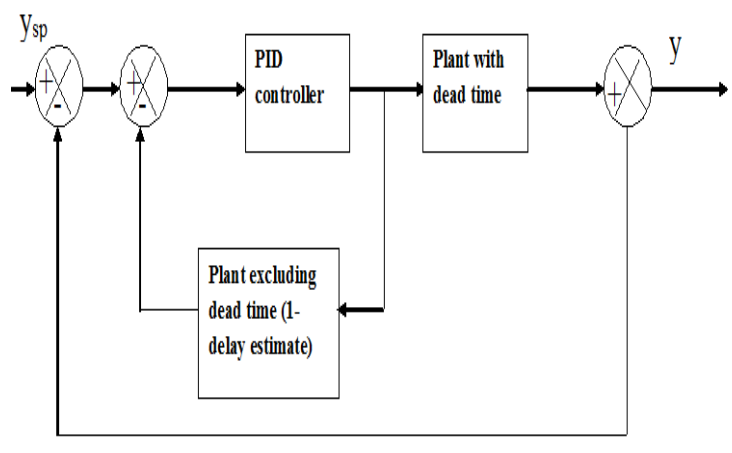

Figure 1. Modified Smith predictor

The PID controller is probably the most widely-used type of feedback controller. PID stands for Proportional-Integral Derivative, referring to the three terms operating on the error signal to produce a control signal. Stability can often be ensured using only the proportional term [4].Proportional-IntegralDerivative (PID) controllers are the most widelyused controllers in process industry, majorly because it is very difficult to improve their cost/advantage ratio [6].

PID controllers have simple structure and tuning methods are widely presented [5]. 


$$
G_{c}(\mathrm{~s})=\mathrm{K}_{\mathrm{p}}\left(1+\frac{1}{\tau_{\mathrm{i}} S}+\tau_{\mathrm{d}} s\right)
$$

\section{METHODOLOGY}

In this present analysis, a second order plus dead time model is selected [1]. After selection of a suitable second order plus dead time process model, controller parameter are calculated to design PID controller using various tuning techniques Ziegler Nichols(ZN), Tyreus Luyben (TL), Pettit and Carr (PC), Chau (C), Bucz (B), modified and Damped Oscillation(DO) method is used [11].

Implement Smith predictor and modified Smith predictor in the system for dead time compensation. Smith is used PID controller for SOPDT process model [9]. Compare the step responses of PID controller for closed loop system design using various tuning techniques with Smith predictor and with modified Smith predictor. Implement Smith predictor and modified smith in the system for dead time compensation.

SOPDT process model is used for many temperature processes. A temperature control loop is selected from literature [10].

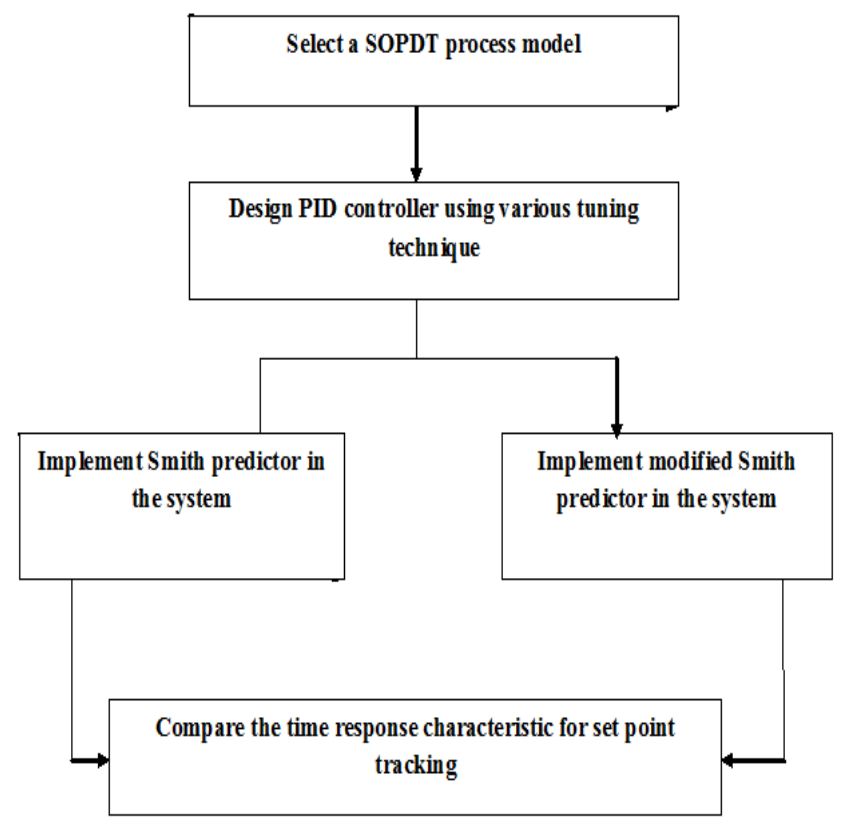

Figure 2. Flow Chart for Systematic Investigation

SOPDT process model is used for many temperature processes [1].

$$
G(s)=\frac{2}{(10 s+1)(5 s+1)} e^{-s}
$$

Where process gain $(\mathrm{k})=2$, Delay time $=1$ second, slow time $\tau_{1}=10$ second, fast time $\tau_{2}=5$ second.

\section{RESULTS AND DISCUSSION}

PID controller has been designed using different tuning techniques and implemented in the closed loop, without Smith Rise time decreases 3.19 second with modified smith predictor.

Comparison of step response of PID controller designed using TL tuning technique without Smith predictor (A4), with modified Smith predictor, with Smith predictor and with Modified Smith predictor for selected SOPDT process model. Simulation is performed to evaluate time response characteristics without Smith predictor with Smith predictor and Modified Smith predictor for SOPDT model.

Figure 3 exhibit the Comparison of step response of PID controller designed using $\mathrm{ZN}$ tuning technique without Smith predictor (A1), with modified Smith predictor (A2) and with Smith predictor (A3) and for selected process model. Settling time is 22 second.

(A5) and with Smith predictor (A6) for selected process model show in figure 4 . Figure 5 indicate the Comparison of step response of PID controller designed using PC1 tuning technique without Smith predictor (A7), with modified Smith predictor (A8) and with Smith predictor (A9) and for selected process model.

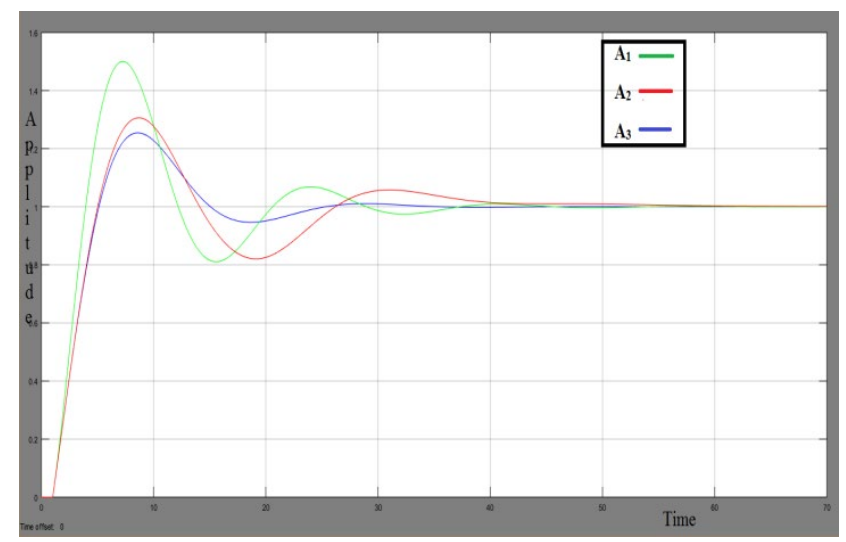

Fig. 3. Comparison of step response of PID controller designed using $\mathrm{ZN}$ tuning technique without Smith predictor (A1), with modified Smith predictor (A2) and with Smith predictor (A3) and for selected process model 


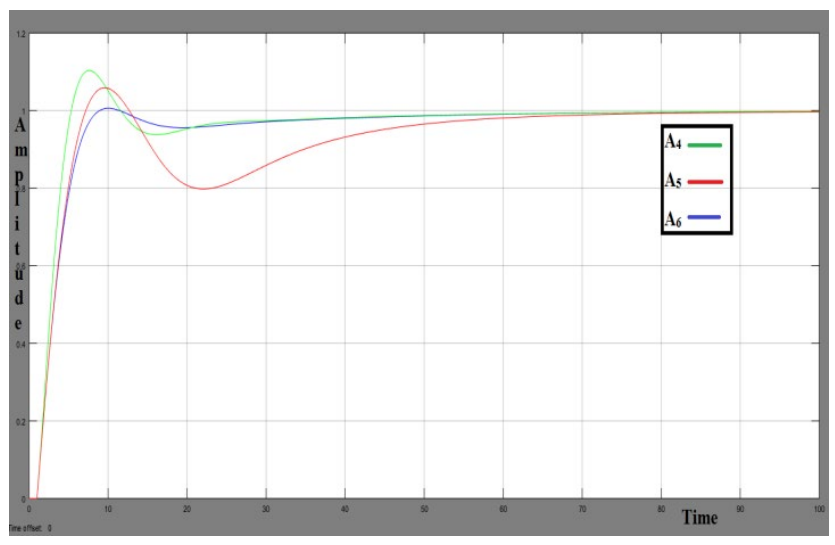

Fig.4. Comparison of step response of PID controller designed using $\mathrm{ZN}$ tuning technique without Smith predictor (A1), with modified Smith predictor (A2) and with Smith predictor (A3) and for selected process model

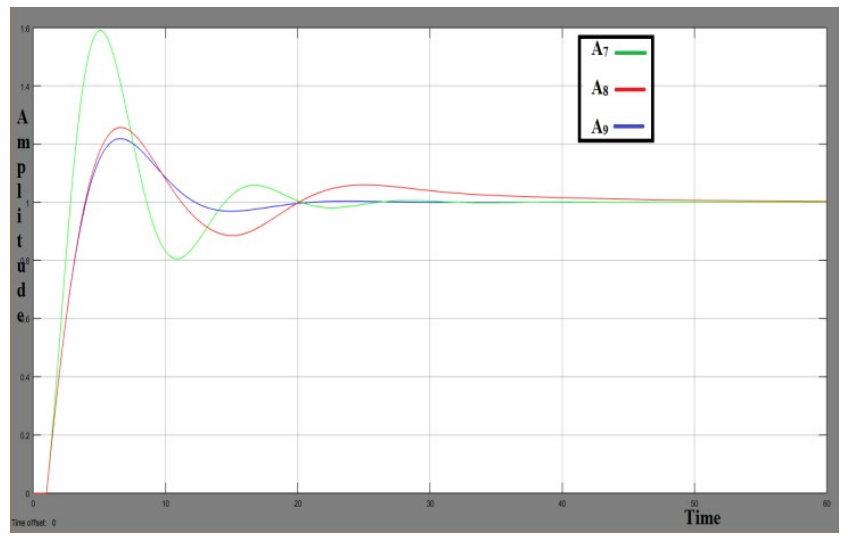

Fig. 5. Comparison of step response of PID controller designed using PC1 tuning technique without Smith predictor (A7), with modified Smith predictor (A8) and with Smith predictor (A9) and for selected process model

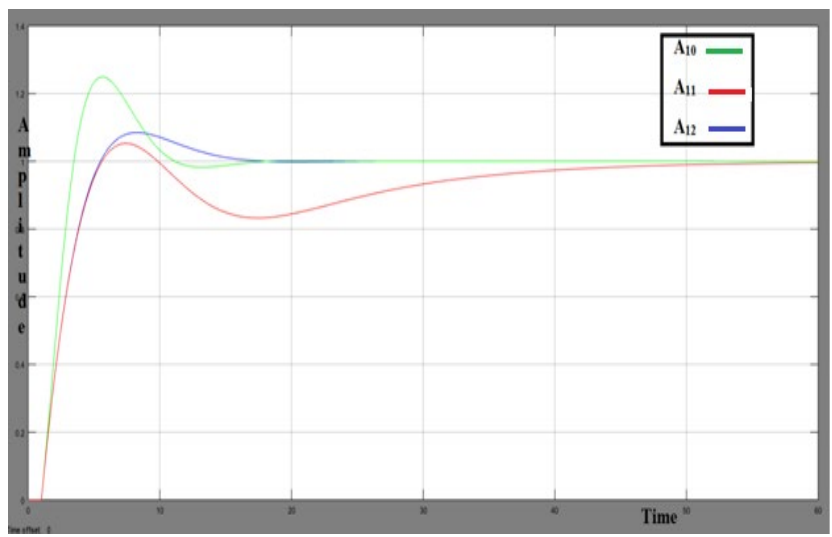

Fig. 6. Comparison of step response of PID controller designed using PC2 tuning technique without Smith predictor (A10), with modified Smith predictor (A11) and with Smith predictor (A12) and for selected process model

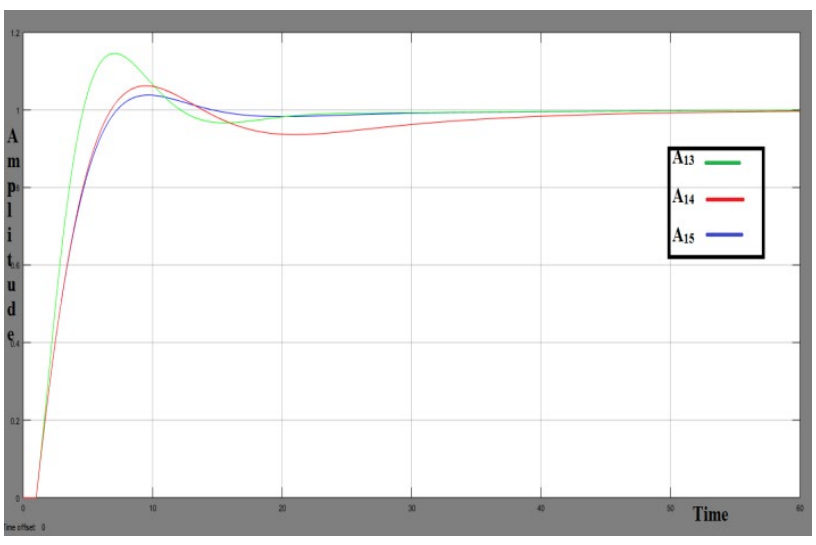

Fig. 7. Comparison of step response of PID controller designed using PC3 tuning technique without Smith predictor (A13), with modified Smith predictor (A14) and with Smith predictor (A15) and for selected process model.

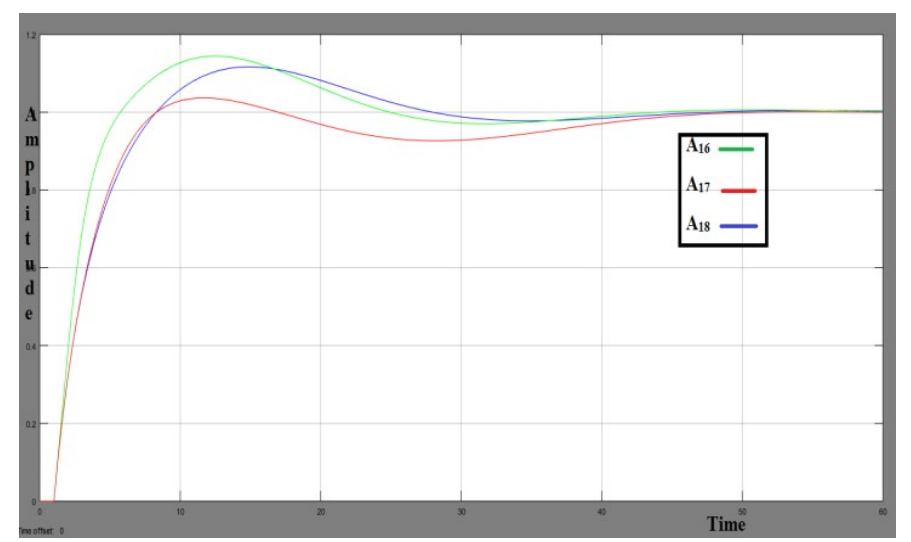

Fig. 8. Comparison of step response of PID controller designed using $\mathrm{Cl}$ tuning technique without Smith predictor (A16), with modified Smith predictor (A17) and with Smith predictor (A18) and for selected process model.

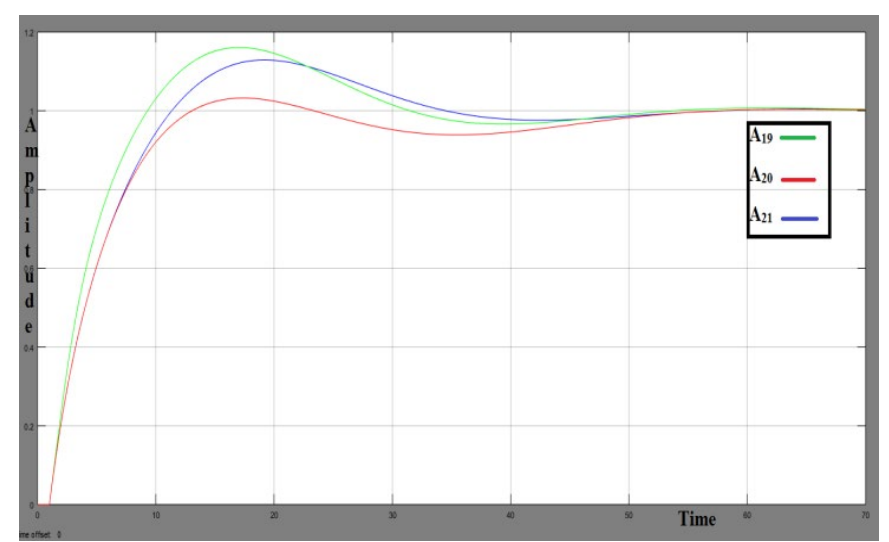

Fig. 9. Comparison of step response of PID controller designed using C2 tuning technique without Smith predictor (A19), with modified Smith predictor (A20) and with Smith predictor (A21) and for selected process model. 


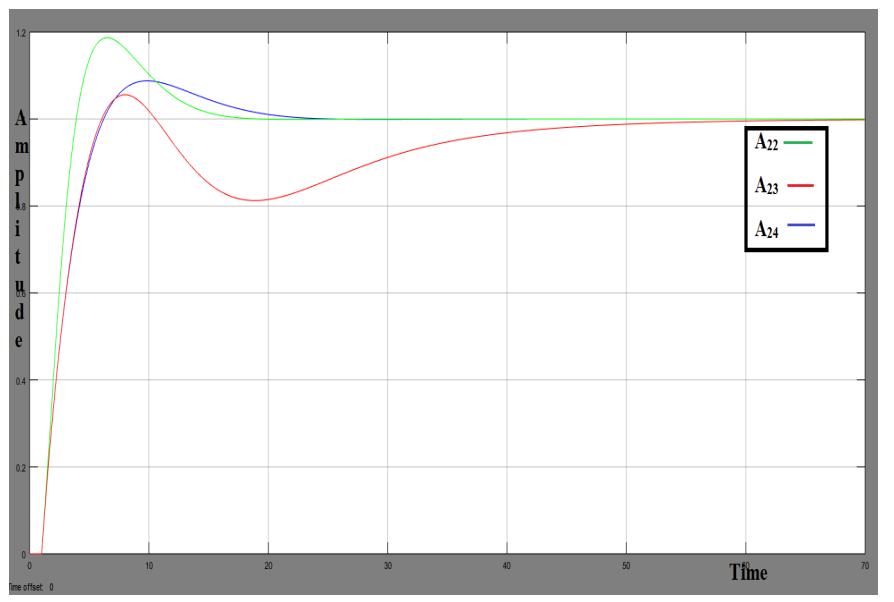

Fig. 10. Comparison of step response of PID controller designed using B1 tuning technique without Smith predictor (A22), with modified Smith predictor (A23) and with Smith predictor (A24) and for selected process model.

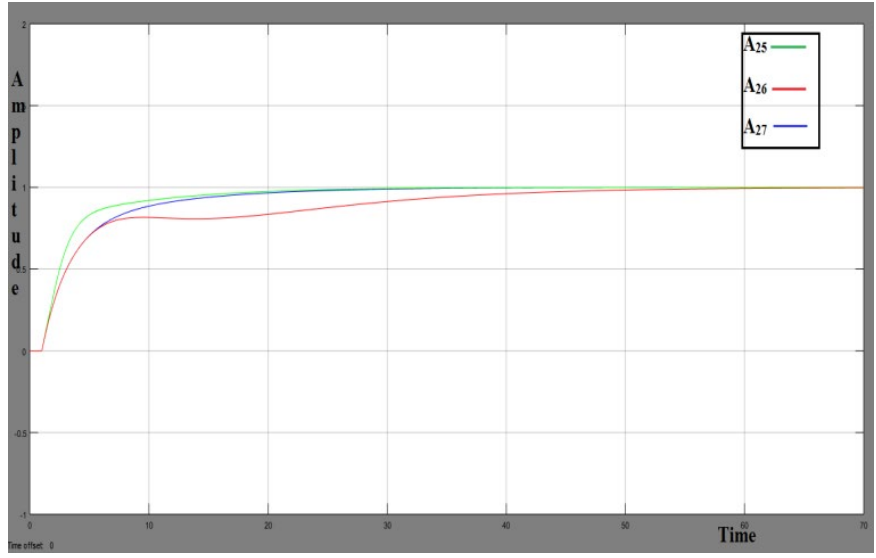

Fig. 11. Comparison of step response of PID controller designed using B2 tuning technique without Smith predictor (A25), with modified Smith predictor (A26) and with Smith predictor (A27) and for selected process model

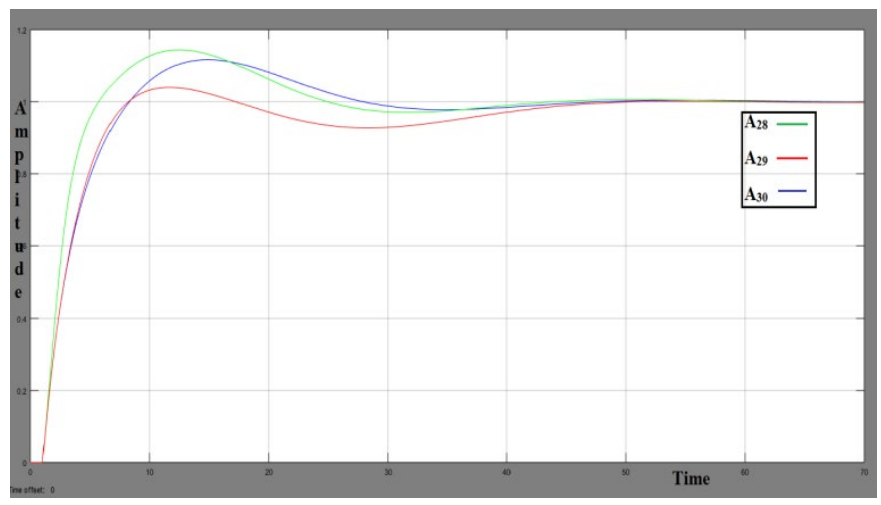

Fig.12. Comparison of step response of PID controller designed using MZ 1 tuning technique without Smith predictor (A28), with modified Smith predictor (A29) and with Smith predictor (A30) and for selected process model

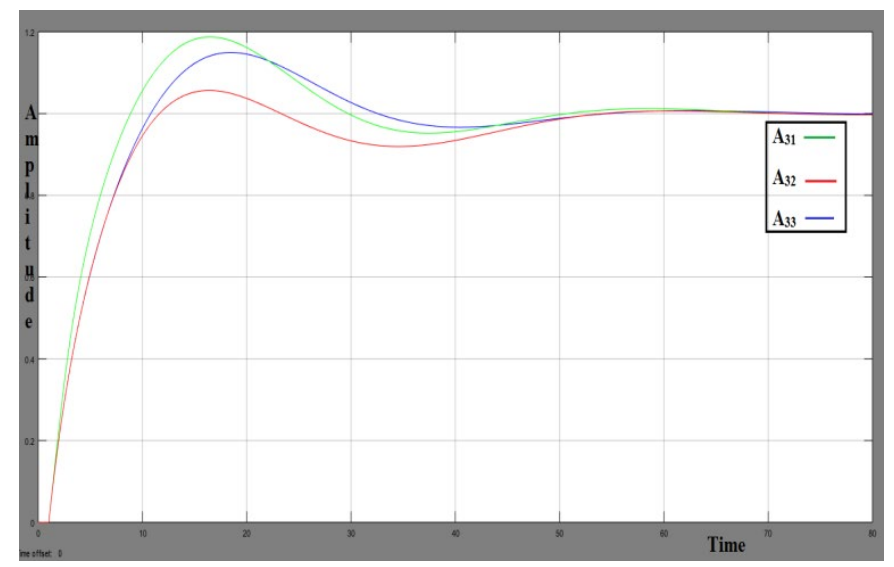

Fig.13. Comparison of step response of PID controller designed using MZ 2 tuning technique without Smith predictor (A31), with modified Smith predictor (A32) and with Smith predictor (A33) and for selected process model

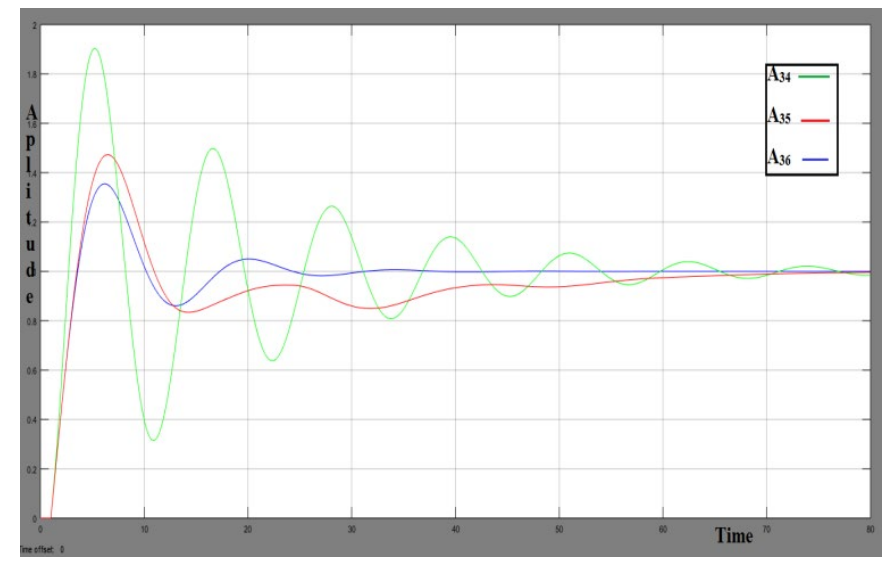

Fig. 14. Comparison of step response of PID controller designed using DS tuning technique without Smith predictor (A31), with modified Smith predictor (A32) and with Smith predictor (A33) and for selected process model

Comparison of step response of PID controller designed using PC2 tuning technique without Smith predictor (A10), with modified Smith predictor (A11) and with Smith predictor (A12) and for selected process model show in figure 6

Figure 7 exhibits the Comparison of step response of PID controller designed using PC3 tuning technique without Smith predictor (A13), with modified Smith predictor (A14) and with Smith predictor (A15) and for selected process model. Comparison of step response of PID controller designed using $\mathrm{C} 1$ tuning technique without Smith predictor (A16), with modified Smith predictor (A17) and with Smith predictor (A18) and for selected process model show in Figure 8. 


\begin{tabular}{|c|c|c|c|c|c|c|c|c|c|}
\hline \multirow{2}{*}{$\begin{array}{l}\text { Tuning } \\
\text { Technique }\end{array}$} & \multicolumn{3}{|c|}{ Without Smith Predictor } & \multicolumn{3}{|c|}{ With Smith Predictor } & \multicolumn{3}{|c|}{ With Modified Smith Predictor } \\
\hline & $\begin{array}{l}\text { Rise } \\
\text { Time }\end{array}$ & $\begin{array}{l}\text { Settling } \\
\text { Time }\end{array}$ & $\begin{array}{c}\text { Max. \% } \\
\text { Overshoot }\end{array}$ & $\begin{array}{l}\text { Rise } \\
\text { Time }\end{array}$ & $\begin{array}{l}\text { Settling } \\
\text { Time }\end{array}$ & $\begin{array}{c}\text { Max. \% } \\
\text { Overshoot }\end{array}$ & $\begin{array}{l}\text { Rise } \\
\text { Time }\end{array}$ & $\begin{array}{c}\text { Settling } \\
\text { Time }\end{array}$ & $\begin{array}{c}\text { Max. \% } \\
\text { Overshoot }\end{array}$ \\
\hline ZN & 2.26 & 34 & 50.1 & 3.32 & 14.8 & 14.9 & 3.19 & 22 & 22.5 \\
\hline $\mathrm{TL}$ & 3.17 & 37.2 & 10.4 & 5.81 & 39.4 & 0 & 4.85 & 38.2 & .618 \\
\hline $\begin{array}{c}\text { PC1 } \\
\text { (under damped) }\end{array}$ & 1.41 & 19.2 & 59.1 & 2.39 & 12.1 & 11.3 & 2.29 & 16.4 & 21.9 \\
\hline $\begin{array}{l}\text { PC2(critically } \\
\text { damped) }\end{array}$ & 1.89 & 10.4 & 24.9 & 3.73 & 13.5 & 3.69 & 3.23 & 12.9 & 8.5 \\
\hline PC3(over damped) & 2.94 & 12.3 & 11.2 & 5.5 & 8.51 & .175 & 4.27 & 11.4 & 3.82 \\
\hline $\mathrm{C} 1$ (small overshoot) & 3.06 & 36.9 & 14.4 & 5.81 & 25.6 & 9.43 & 5.1 & 36.6 & 11.7 \\
\hline $\begin{array}{l}\text { C2(without } \\
\text { overshoot) }\end{array}$ & 6.04 & 46.4 & 16.1 & 8.35 & 32 & 10.4 & 7.65 & 45.8 & 12.9 \\
\hline B1(over shoot $\leq 20$ ) & 2.18 & 14.3 & 18.7 & 4.28 & 18.4 & 5.22 & 3.71 & 17.1 & 8.78 \\
\hline $\mathrm{B} 2\left(\mathrm{t}_{\mathrm{c}} \geq 13 / \omega\right)$ & 6.8 & 22.1 & 0 & 11.2 & 25.4 & 0 & 9.69 & 23.6 & 0 \\
\hline $\begin{array}{c}\text { MODIFIED } \\
\text { ZN(small overshoot) }\end{array}$ & 3.06 & 36.9 & 14.4 & 5.81 & 25.7 & 9.43 & 5.11 & 36.6 & 11.7 \\
\hline $\begin{array}{l}\text { MODIFIED ZN(no } \\
\text { overshoot) }\end{array}$ & 5.8 & 45.9 & 18.8 & 7.97 & 44.8 & 12.1 & 7.35 & 46.2 & 15 \\
\hline Damped Oscillation & 1.33 & 75 & 90.8 & 1.97 & 16.6 & 19.1 & 2.02 & 21.9 & 35.7 \\
\hline
\end{tabular}

Comparison of step response of PID controller designed using C2 tuning technique without Smith predictor (A19), with modified Smith predictor (A20) and with Smith predictor (A21) and for selected process model is given by figure 9.Figure 10 exhibits the Comparison of step response of PID controller designed using B1 tuning technique without Smith predictor (A22), with modified Smith predictor (A23) and with Smith predictor (A24) and for selected process model.

Comparison of step response of PID controller designed using B2 tuning technique without Smith predictor (A25), with modified Smith predictor (A26) and with Smith predictor (A27) and for selected process model is given by figure 11 . Fig 12 show the Comparison of step response of PID controller designed using MZ 1 tuning technique without Smith predictor (A28), with modified Smith predictor (A29) and with Smith predictor (A30) and for selected process model
Comparison of step response of PID controller designed using $\mathrm{MZ} 2$ tuning technique without Smith predictor (A31), with modified Smith predictor (A32) and with Smith predictor (A33) and for selected process model is given by figure 13.rise time is reduced 7.35 second which is decreases with modified Smith predictor. Figure 14 exhibits the Comparison of step response of PID controller designed using DS tuning technique without Smith predictor (A31), with modified Smith predictor (A32) and with Smith predictor (A33) and for selected process model.

\section{CONCLUSION}

In present analysis PID controller has been designed using various tuning techniques and implemented in a closed loop, with Smith predictor, with modified smith predictor and without smith predictor for dead time compensation for SOPDT process model. In all of the cases rise time has increased with the implementation of Smith 
Predictor while Maximum Percentage Overshoot decreased. In most of the cases settling time has also decreased with the use of Smith Predictor.

Modified Smith predictor decreases rise time in most of the cases but settling time and maximum percentage overshoot increases. So effect of dead time is compensated by using modified Smith predictor. But if less settling time is one of the control objective apart from dead time compensation Smith Predictor is more advantageous.

\section{REFERENCES}

[1] D. E. Seborg, T. F. Edgar, D. A. Mellichamp, F. J. Doyle "Process Dynamics and Control", 3rd Edition, Wiley Publications, United States.

[2] N.Abe and K. Ymamaka, "Smith Predictor control and Internal Model Control - A tutorial" proceeding of SICS Annual conference, Fukui, Japan, 4-6 August, 1383-1387, 2003.

[3] Visioli, "Research trends for PID controllers," Acta Polytechnica, Vol. 52, No. 5, pp. 144-150, 2012.

[4] J. Shi, W. S. Lee, "Set point response and disturbance rejection tradeoff for Second-Order plus Dead Time Processes," 5th Asian Conference, pp. 881-887, 2004.

[5] Mayank Chaturvedi, Pradeep Juneja, "Effect of dead time approximation on controller performance designed for a second order delayed Model" proceedings of International

[6] Wen Tan a, Jizhen Liu a, Tongwen Chenb, Horacio J. Marquez "Comparison of some well-known PID tuning formulas" Computers and Chemical Engineering 1416-1423, 2006.

[7] C.C. Hang, "Smith Predictor and its Modification," Control Systems, Robotics and Automation, Vol. 2 pp. 119-126,

[8] Prateeksha Chauhaan, Pradeep K. Juneja, Mayank Chaturvedi, "Controller design and its performance analysis for a delayed process model" International Conference on Advances in Computing, Communications and Informatics (ICACCI) pp. 859 $-862,2014$.

[9] Sigurd Skogestad,' Simple analytic rules for model reduction and PID controller tuning", Journal of Process Control Vol. 13,pp. 291-309,2003.

[10] O'Dwyer, "PI and PID controller tuning rules for time delay processes: a summary Technical Report" AOD-00-01, 1stEdition, Dublin, Ireland, 15 May 2000

[11] Panda "Introduction to PID Controllers Theory, Tuning and Application To Frontier Areas" InTech publication. 